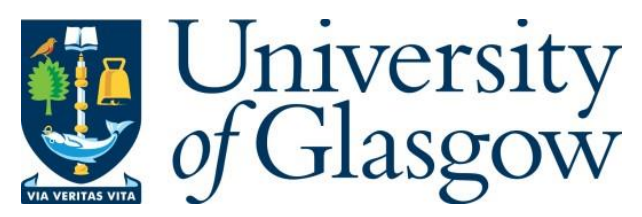

Chandra, T. and Kirschner, K. (2016) Chromosome organisation during ageing and senescence. Current Opinion in Cell Biology, 40, pp. 161-167.

There may be differences between this version and the published version. You are advised to consult the publisher's version if you wish to cite from it.

http://eprints.gla.ac.uk/138657/

Deposited on: 25 August 2017

Enlighten - Research publications by members of the University of Glasgow http://eprints.gla.ac.uk 


\section{Chromosome organisation during ageing and senescence}

Tamir Chandra ${ }^{1^{*}}$ and Kristina Kirschner ${ }^{2^{*}}$

1 Epigenetics Programme, The Babraham Institute, Cambridge CB22 3AT, UK; The Wellcome Trust Sanger Institute, Cambridge CB10 1SA, UK. Electronic address: tamir.chandra@babraham.ac.uk.

2 Cambridge Institute for Medical Research, Wellcome Trust/MRC Stem Cell Institute and Department of Haematology, University of Cambridge, Hills Road, Cambridge CB2 OXY, UK.; Electronic address: kk429@cam.ac.uk

* Corresponding author

Highlights

- Acute and chronic cellular stress models show distinct nuclear phenotypes

- Heterochromatin relaxes in acute and chronic cellular stress

- Heterochromatin relaxation causes the activation of repeat elements

- Mechanisms specific to acute stress might block repeat activation

Abstract

Acute cellular stress caused by oncogene activation or high levels of DNA damage can engage a tumour suppressive response, which can lead to cellular senescence. Chronic cellular stress evoked by low levels of DNA damage or telomere erosion is involved in the ageing process. In oncogene induced senescence in fibroblasts, a dramatic rearrangement of heterochromatin into foci and accumulation of constitutive heterochromatin is well documented. In contrast, a loss of heterochromatin has been described in replicative senescence and premature ageing syndromes. The distinct nuclear phenotypes that accompany the stress response highlight the differences between acute and chronic stress models, and this review will address the differences and similarities between these models with a focus on chromosome organisation and heterochromatin.

Introduction

Cellular senescence describes the response of a cell to cellular stress, which is mostly linked to genotoxic stress or DNA damage [1-5]. However, the senescence stress response can be very heterogeneous depending on the way the stress is induced. A useful working model of classifying stress responses has been by dividing them into models where damage accumulates slowly over months and years, such as in replicative senescence; and acute stress models that evoke a senescence response within hours, such as oncogene induced senescence (OIS) (Figure 1). The exact event downstream of oncogene activation triggering the senescence response is still debated. However, oncogene activation can lead to complete senescence of a fibroblast population in 48 hours in the presence of ectopic telomerase[6]. This distinction between chronic and acute stress models allows comparisons to stress situations that might not be considered senescence models per se, such as premature ageing (progeroid) syndromes, which can be considered chronic stress situations based on their slow kinetics. In addition there are many lines of evidence linking progeroid syndromes and cellular senescence[7-9]. Werner syndrome is an adult onset premature ageing syndrome caused by mutations in the Wrn DNA helicase gene, leading to increased levels of DNA 
damage in patients [10]. Mesenchymal stem cells (MSCs) derived from Wrn null (-/-) embryonic stem cells display a pronounced senescence phenotype upon serial passaging in vitro and in stem cell transplantation experiments [10]. Hutchinson-Gilford progeria syndrome (HGPS or progeria) is caused by a mutation in the lamin A (LMNA) gene and manifests in early childhood [11,12]. Cells from HGPS patients show many hallmarks of senescence such as elevated DNA damage levels and telomere attrition $[9,13,14]$. As such, chronic stress situations have mostly been studied in the context of cellular ageing, whereas acute models have served to understand the tumour suppressive role of senescence [15]. While the distinction between stress situations and phenotype seems useful, there have been interesting observations suggesting a crosstalk between the two (indicated by the dashed arrows in Fig 1). One example of such cross-talk is a recently discovered barrier to oncogenic transformation in progeroid cells [16].

The way that senescence or chronic cellular stress contributes to organismal ageing is not fully understood. However, there is evidence for at least two independent scenarios in which senescence has been implicated in ageing. The p53 and INK4a/ARF loci have long been associated with senescence and there is emerging evidence that both loci are implicated in organismal ageing via deregulation of the stem cell pool [15]. Chronic hyperactivation of p53 was shown to result in reduced proliferation of haematopoietic stem cells (HSC) upon stress through transplantation experiments [17]. However, there is also evidence that the role of p53 activation in ageing might be more complex [18]. With age, increased DNA damage and p16Ink4a expression leads to a reduction in HSC cell cycle activity $[19,20]$. Environmental stress or cells reaching their replicative life span stimulate stem cells to replenish the pool of somatic cells. As an organism ages, or in chronic stress situations, the stem cell pool is functionally diminished and therefore unable to reconstitute tissue (Figure 1). Slow kinetics characterise this process, with the rate of senescence determining the rate of stem cell exhaustion.

Another way senescence has been implicated in ageing is through its senescence associated secretory phenotype (SASP), which is characterised by secretion of cytokines and matrixmetalloproteases [21-23]. SASP functions in two ways: it reinforces the senescence response in neighbouring cells through its secretome and it recruits immune cells to clear senescent cells. However, senescent cells that are not cleared can contribute to age-related pathologies over time, most likely through chronic inflammation triggered by their SASP response (Figure 1)[15]. Indeed it seems that some age related pathologies can be alleviated by eliminating p16Ink4a senescent cells from tissue [24].

Nuclear organisation and constitutive heterochromatin $(\mathrm{cHC})$

The distinct nuclear phenotypes that accompany the stress response highlight the differences between acute and chronic stress models, and this review will address the differences and similarities between these models with a focus on chromosome organisation and heterochromatin. Although a role for $\mathrm{CHC}$ and the nuclear architecture in cellular ageing was first proposed nearly 20 years ago [25], the concept received spotlight attention from 2003 due to the discovery that HGPS is caused by mutations in the LMNA gene $[11,12]$. In the same year a new nuclear phenotype was described in cells undergoing oncogene-induced senescence and other forms of acute stress and was named for its spotty pattern of heterochromatic domains: senescence associated heterochromatic foci (SAHF) (Figure 2) [26]. Mapping heterochromatic markers revealed striking differences in the 
fate of $\mathrm{cHC}$ marks: a loss in chronic stress situations[27-29] and an accumulation during stress induced senescence [26].

Acute stress: SAHF positive cells

SAHF formation in senescent cells is a striking nuclear phenotype (see Figure 2), which leads to the formation of 4',6-diamidino-2-phenylindole (DAPI) intense foci. Studies using chromosome painting have revealed that each focus consists of exactly one chromosome [30-32]. The core area of the SAHF is enriched with markers for constitutive heterochromatin such as Histone 3 lysine 9 trimethylation (H3K9me3), heterochromatin protein 1 (HP1) and macro Histone $2 \mathrm{~A}(\mathrm{mH} 2 \mathrm{~A})[26,33]$, whereas the SAHF periphery is enriched with the facultative heterochromatin mark Histone 3 lysine 27 trimethyl (H3K27me3) [32]. Euchromatic regions can be found outside the DAPI intense focus and the H3K27me3 ring. Figure 2 depicts the overall architecture of SAHF [32,34]. The geometry of chromatin domains therefore seems inversed when compared to proliferating cells, where the heterochromatin is found in the periphery of chromosomal territories [35]. Initial theories surrounding the function of SAHF speculated that SAHF might be the result of de novo heterochromatin formation in euchromatic areas, for example for the silencing of cell cycle genes. However, through ChIP-seq and DNA labelling studies it has been shown that the heterochromatic regions forming the core of the SAHF are not de novo heterochromatic regions, but are the same regions that form the heterochromatin in proliferating cells, at least on a global level [32]. In agreement with this finding, SAHF positive cells show a loss of heterochromatin at the nuclear periphery under the electron microscope (Narita) and SAHF are found away from the nuclear lamina $[32,36]$. The nuclear lamina forms a mesh that supports the structural integrity and shape of the nucleus. In addition it serves as scaffold for coordinating other key events within the nucleus such as transcription and DNA replication $[37,38]$. The key structural components of the lamina are Lamins $\mathrm{A} / \mathrm{C}$ and $\mathrm{B}$ and the loss of Lamin B1 (LMNB1) in senescence might be responsible for the detachment from the nuclear periphery $[36,39,40]$. It has also been shown that LMNB1 reduction is a requirement, although not sufficient, for SAHF formation [36]. While global mapping of LMNB1 through ChIP-seq studies confirmed a global loss of LMNB1 on heterochromatic areas in senescence, it also identified a small region of the genome (2\%) with increased levels of LMNB1 [36]. Interestingly, fluorescence in situ hybridisation (FISH) studies suggested that these regions with increasing LMNB1 levels show a tendency to reposition towards the nuclear lamina and might be involved in gene silencing in senescence [36,41]. In OIS, SAHF formation and destabilisation of the lamina are completed within hours/days and how this dynamic change occurs is not well understood, however a recent study has shown an active and specific involvement of the autophagy machinery in turning over the nuclear lamina [42].

Chronic stress: Replicative senescence and progeroid syndromes

Dramatic changes to the nuclear lamina and nuclear morphology are also the hallmark of HGPS. The expression of mutant LMNA, called progerin, prevents an important step in the processing of LMNA from occurring and leads to a permanently farnesylated form of LMNA, which seems to be immobilised at the nuclear periphery [43]. How progerin causes changes in the lamina and how these changes relate to nuclear defects is not entirely understood and is discussed in more depth in other review articles [44,45]. However, the repositioning of heterochromatin from the nuclear periphery is also seen in HGPS and seems to be achieved through independent mechanisms: by 
LMNA mutation in HGPS and LMNB1 downregulation in SAHF positive cells. In addition, the progeroid nucleus shows a global loss of heterochromatic marks, such as H3K9me3 and H3K27me3, and similar reductions have been observed in replicative senescence and in cells from older individuals $[27,28,46]$. A loss of heterochromatic marks has also been noted in a Werner syndrome model, WRN-/- MSCs [10]. The loss of heterochromatic marks in HGPS, Wrn knockout cells and replicative senescence is in stark contrast to the perceived increase in heterochromatin based on the DAPI intensity in SAHF positive cells and the increase in heterochromatin proteins (HP1) on senescent chromatin [26].

Heterochromatin relaxation in chronic and acute stress responses

Heterochromatin can be defined either by its enrichment by marker proteins or by its structural properties. One approach that maps the structural properties of the genome is the Hi-C technology [47], which has been used on SAHF positive ( $86 \%$ ) senescent cells to shed light on the seemingly contradictory roles of heterochromatin in chronic stress models and SAHF formation [41]. In proliferating cells, constitutive heterochromatic regions display the highest amount of internal contacts, measured by $\mathrm{Hi}-\mathrm{C}$, when compared to other regions in the genome. $\mathrm{Hi}-\mathrm{C}$ data in SAHF positive cells show a loss of local interactions within constitutive heterochromatic domains. These domains can be further identified by their low GC content and their lamin association in proliferating cells $[41,48]$. FISH experiments measuring the compaction of changing heterochromatin suggest that the loss of local interactions leads to a relaxation of these domains. This observation is contrary to the previously held view of enhanced heterochromatin in SAHF formation. Despite the increase in heterochromatic marks in SAHF positive cells, the heterochromatin seems to relax on a structural level. Notably, in Hi-C data from HGPS, heterochromatic domains show a similar tendency as observed for the SAHF positive cells [41,49]. In addition late passage HGPS cells show a global loss of genome structure, such as the partitioning into active and inactive domains [49]. SAHF cells also show long-range clustering of large $\mathrm{CHC}$ domains along the chromosome, which may reflect the clustering of constitutive heterochromatin in the core of the SAHF. Indeed, HGPS cells, devoid of SAHF formation do not show clustering of HC domains (see Figure 3) [41].

While the observation that heterochromatin relaxes in OIS was unexpected it aligned well with the observation that high mobility group A (HMGA) proteins, which stabilise a more open chromatin conformation, are among the most dramatically enriched proteins in oncogene-induced senescent chromatin reaching an abundance similar to core histones [50,51]. HMGA1/2 knockdown almost completely blocks SAHF formation. Knockdown in an OIS population reduces the number of SAHF positive cells suggesting that this plays a role in SAHF maintenance $[32,50]$. Their described function as architectural proteins and abundance of HMGA1/2 proteins suggest that they might be structural components of SAHF. Ectopic HMGA1/2 expression works synergistically with LMNB1 knockdown to induce some de novo SAHF formation [36]. HMGA1/2 might compete with the linker histone $1(\mathrm{H} 1)$ for the same niche in the genome, as both proteins preferentially bind to the minor groove of ATrich DNA [51]. Notably, H1 is absent on senescent chromatin [30]. To our knowledge HMGA has not been studied in chronic stress situations, such as replicative senescence or progeria. Other factors involved in SAHF formation including histone chaperones and histone variants have been reviewed recently and go beyond the scope of this review $[52,53]$. 
The observation that heterochromatin might be relaxing in senescence aligns well with the finding that normally heterochromatic alpha-satellite and satellite II repeats relax in senescence. This process is termed senescence associated distension of satellites (SADS) and has been described as an early event in a variety of senescence models and HGPS cells $[54,55]$. In addition, extensive transcription and retro-transposition of LINE elements has been described in replicative senescence, partially mediated through downregulation of SIRT6 [56-59]. However no upregulation of repeats has been reported for acute senescence systems as resulting in a high number of SAHF positive cells. In addition our preliminary data do not show LINE activation in OIS cells.

\section{Discussion}

In this review we compare the different nuclear phenotypes that accompany chronic and acute cellular stress responses, such as replicative senescence and progeria (chronic), and oncogene induced senescence (acute). While these models agree on a number of key insights, the role of constitutive heterochromatin ( $\mathrm{cHC}$ ) had been controversial, with chronic stress models showing a reduction and acute stress models showing an increase in $\mathrm{cHC}$ based on analysis of marker proteins [27-29]. Recent work has examined changes in nuclear architecture by genome-wide sequencing (using $\mathrm{Hi}-\mathrm{C}$ ), which has led to the finding that architectural changes are conserved between Hutchinson-Gilford progeria and OIS, resulting in a relaxation of the $\mathrm{CHC}$ compartment in both [41]. Another recent study found a loss of $\mathrm{cHC}$ in Werner Syndrome, an adult onset progeria so far associated with genome instability[10]. The common architectural changes in the $\mathrm{cHC}$ compartment, achieved through independent mechanisms (for example dysfunctional LMNA in progeria and LMNB1 degradation in OIS) point towards a functional role for the relaxation of the $\mathrm{CHC}$ compartment in cellular stress and ageing. However, we currently do not know what that function might be. One possible scenario was proposed in a recent study showing the activation and transposition of LINE elements in replicative senescence. These retrotransposition events may be a mechanism to induce or amplify a DNA damage signalling cascade, thereby inducing or reinforcing senescence $[56,57]$.

In contrast to $\mathrm{CHC}$ relaxation being found in chronic and acute stress, spatial clustering of heterochromatic regions and an upregulation of heterochromatin proteins seems SAHf specific, suggesting a second step unique to acute stress induced senescence (see Figure 3). A possible scenario that would also explain the difference in the upregulation of heterochromatic markers, such as HP1 and macroH2A, could be that SAHF formation acts as a compensatory mechanism to maintain or re-silence repetitive elements (Figure 4). More precisely the relaxation of the $\mathrm{CHC}$ domains could trigger the rise of chromatin bound HP1 and macroH2A, which might result in the spatial clustering of the $\mathrm{CHC}$ domains to maintain heterochromatic silencing and which might result in the SAHF phenotype.

Acknowledgments: We thank Dr. Laura Blackburn for critical reading of the manuscript. KK is supported through a Bloodwise grant held by A.R. Green (grant ref. 13003). TC is equally funded through the Wellcome Trust Sanger and Babraham Institutes.

Figure 1: Chronic (green) and acute stress (red) induced DNA damage leads to different cell fate choice. In the schematic the circles represent the two different types of stress with selected 
underlying causes. Chronic stress is characterised through slow kinetics and a gradual accumulation of damage, which has mostly been studied in the context of ageing on the cellular and organismal level (left hand side of diagram). In contrast, oncogene activation and subsequent hyperproliferation or high level DNA damage activate an acute stress response, leading to senescence as part of the organismal tumour suppressive response (right hand side of the diagram). However, crosstalk between the two models exist (dashed arrows), for example through progeria mediated resistance to transformation by inhibiting oncogenic dedifferentiation.

Figure 2: Senescent chromosomes form senescence associated heterochromatic foci (SAHF), which show a segregation and clustering of different chromatin types. On the left a schematic of a SAHF positive nucleus is drawn as it would appear after being stained by DAPI (black). Each of the foci represents a SAHF, which represents an individual chromosome (Chr). One of the foci is schematically enlarged to visualise the multilayer chromatin structure found in SAHF (center). To the right, an immuno-fluorescently stained SAHF is shown for comparison. The chromatin types are represented here through histone modifications. The core of the SAHF is enriched in H3K9me3 (green, constitutive heterochromatin). A ring at the periphery of the SAHF is enriched in H3K27me3 (red, facultative heterochromatin/Polycomb silencing). Active euchromatin can be found outside the SAHF and is shown by H3K36me3 (blue).

Figure 3: A two-step model for heterochromatin dynamics in chronic and acute cellular stress. Depicted on the left is the nuclear periphery of a proliferating cell. Lamin associated domains (LADs), consist of compacted constitutive heterochromatin (cHC, green) and are attached to the nuclear lamina (purple). H3K27me3 (red) often flanks the $\mathrm{CHC}$ in LADs. Euchromatic regions are shown in blue. As an initial stress response in acute (for example oncogene activation) and chronic stress (for example progeria and replicative senescence) LADs detach from the lamina (purple) and it comes to a relaxation of the heterochromatin (green). In this model, Step 1 presents the endpoint for chronic stress response, whereas it might be an intermediate step for SAHF forming cells. Step 2 shows the spatial clustering of $\mathrm{cHC}$ (green domains coming together) as suggested for SAHF positive cells. A set of unexplored regions in the genome (2\%) gain LMNB1 and move towards the periphery in SAHF positive cells (yellow, LAD?).

Figure 4: Tentative model for the involvement of SAHF formation in compensating heterochromatin relaxation and repeat activation. Decompaction of heterochromatic regions involved in the silencing of satellite repeats has been suggested as an early event for all types of senescence. In addition LINE transcriptional activation of LINE elements has been shown for replicative senescence, but has not been found in acute stress, such as oncogene induced senescence. The upper nucleus in the figure depicts the detached and decompacted heterochromatin, including the activation of repeats during the initial stress response (green, see also Figure 3). The lower nucleus shows a speculative role for SAHF formation in compensating the repeat activation. SAHF formation is represented here through the influx of heterochromatin associated markers (heterochromatin proteins and the macroH $2 \mathrm{~A}$ histone shown here in purple) and the spatial clustering of heterochromatic regions (green).

1. Campisi J, d'Adda di Fagagna F: Cellular senescence: when bad things happen to good cells. Nat. Rev. Mol. Cell Biol. 2007, 8:729-40. 
2. Kuilman T, Michaloglou C, Mooi WJ, Peeper DS: The essence of senescence. Genes Dev. 2010, 24:2463-79.

3. Salama R, Sadaie M, Hoare M, Narita M: Cellular senescence and its effector programs. Genes Dev. 2014, 28:99-114.

4. Pawlikowski JS, Adams PD, Nelson DM: Senescence at a glance. J. Cell Sci. 2013, 126:4061-7.

5. Muñoz-Espín D, Serrano M: Cellular senescence: from physiology to pathology. Nat. Rev. Mol. Cell Biol. 2014, 15:482-96.

6. Jeanblanc M, Ragu S, Gey C, Contrepois K, Courbeyrette R, Thuret J-Y, Mann C: Parallel pathways in RAF-induced senescence and conditions for its reversion. Oncogene 2012, 31:3072-85.

7. Cao K, Blair CD, Faddah DA, Kieckhaefer JE, Olive M, Erdos MR, Nabel EG, Collins FS: Progerin and telomere dysfunction collaborate to trigger cellular senescence in normal human fibroblasts. J. Clin. Invest. 2011, 121:2833-44.

8. Soria-Valles C, Osorio FG, Gutiérrez-Fernández A, De Los Angeles A, Bueno C, Menéndez P, Martín-Subero JI, Daley GQ, Freije JMP, López-Otín C: NF-KB activation impairs somatic cell reprogramming in ageing. Nat. Cell Biol. 2015, 17:1004-13.

9**. Chojnowski A, Ong PF, Wong ESM, Lim JSY, Mutalif RA, Navasankari R, Dutta B, Yang H, Liow YY, Sze SK, et al.: Progerin reduces LAP2 $\alpha$-telomere association in Hutchinson-Gilford progeria. Elife 2015, 4.

9** Here loss of heterochromatin and proliferation defects in Hutchinson- Gilford progeria syndrome is linked to loss of interactions of the lamina with telomeres.

10**. Zhang W, Li J, Suzuki K, Qu J, Wang P, Zhou J, Liu X, Ren R, Xu X, Ocampo A, et al.: A Werner syndrome stem cell model unveils heterochromatin alterations as a driver of human aging. Science 2015, 348:1160-3.

10** The premature ageing phenotype of Werner syndrome patients has been mostly associated with their increased levels in DNA damage. This paper uncovers a role for Werner protein in maintenance of heterochromatin stability. A connection between heterochromatin disorganisation and premature ageing in mesenchymal stem cells is established.

11. Eriksson M, Brown WT, Gordon LB, Glynn MW, Singer J, Scott L, Erdos MR, Robbins CM, Moses TY, Berglund P, et al.: Recurrent de novo point mutations in lamin A cause Hutchinson-Gilford progeria syndrome. Nature 2003, 423:293-8.

12. De Sandre-Giovannoli A, Bernard R, Cau P, Navarro C, Amiel J, Boccaccio I, Lyonnet S, Stewart $\mathrm{CL}$, Munnich $\mathrm{A}$, Le Merrer $\mathrm{M}$, et al.: Lamin a truncation in Hutchinson-Gilford progeria. Science 2003, 300:2055.

13. Liu B, Wang J, Chan KM, Tjia WM, Deng W, Guan X, Huang J, Li KM, Chau PY, Chen DJ, et al.: Genomic instability in laminopathy-based premature aging. Nat. Med. 2005, 11:780-5. 
14. Decker ML, Chavez E, Vulto I, Lansdorp PM: Telomere length in Hutchinson-Gilford progeria syndrome. Mech. Ageing Dev. 2009, 130:377-83.

15. Van Deursen JM: The role of senescent cells in ageing. Nature 2014, 509:439-46.

16*. Fernandez P, Scaffidi P, Markert E, Lee J-H, Rane S, Misteli T: Transformation resistance in a premature aging disorder identifies a tumor-protective function of BRD4. Cell Rep. 2014, 9:248-60.

16* Hutchinson-Gilford progeria (HGPS) patients are protected from carcinogenesis despite elevated levels of DNA damage. This paper describes for the first time a mechanism of transformation resistance in cells from HGPS patients.

17. Dumble M, Moore L, Chambers SM, Geiger H, Van Zant G, Goodell MA, Donehower LA: The impact of altered p53 dosage on hematopoietic stem cell dynamics during aging. Blood 2007, 109:1736-42.

18. Baker DJ, Weaver RL, van Deursen JM: p21 both attenuates and drives senescence and aging in BubR1 progeroid mice. Cell Rep. 2013, 3:1164-74.

19. Rossi DJ, Bryder D, Seita J, Nussenzweig A, Hoeijmakers J, Weissman IL: Deficiencies in DNA damage repair limit the function of haematopoietic stem cells with age. Nature 2007, 447:725-9.

20. Janzen V, Forkert R, Fleming HE, Saito Y, Waring MT, Dombkowski DM, Cheng T, DePinho RA, Sharpless NE, Scadden DT: Stem-cell ageing modified by the cyclin-dependent kinase inhibitor p16INK4a. Nature 2006, 443:421-6.

21. Coppé J-P, Patil CK, Rodier F, Sun Y, Muñoz DP, Goldstein J, Nelson PS, Desprez P-Y, Campisi J: Senescence-associated secretory phenotypes reveal cell-nonautonomous functions of oncogenic RAS and the p53 tumor suppressor. PLoS Biol. 2008, 6:2853-68.

22. Acosta JC, O'Loghlen A, Banito A, Guijarro M V, Augert A, Raguz S, Fumagalli M, Da Costa M, Brown $C$, Popov $N$, et al.: Chemokine signaling via the CXCR2 receptor reinforces senescence. Cell 2008, 133:1006-18.

23. Acosta JC, Banito A, Wuestefeld T, Georgilis A, Janich P, Morton JP, Athineos D, Kang T-W, Lasitschka $\mathrm{F}$, Andrulis $\mathrm{M}$, et al.: A complex secretory program orchestrated by the inflammasome controls paracrine senescence. Nat. Cell Biol. 2013, 15:978-90.

24. Baker DJ, Wijshake T, Tchkonia T, LeBrasseur NK, Childs BG, van de Sluis B, Kirkland JL, van Deursen JM: Clearance of p16Ink4a-positive senescent cells delays ageing-associated disorders. Nature 2011, 479:232-6.

25. Villeponteau B: The heterochromatin loss model of aging. Exp. Gerontol. 32:383-94.

26. Narita M, Nũnez S, Heard E, Narita M, Lin AW, Hearn SA, Spector DL, Hannon GJ, Lowe SW: Rb-mediated heterochromatin formation and silencing of E2F target genes during cellular senescence. Cell 2003, 113:703-16. 
27. Tsurumi A, Li WX: Global heterochromatin loss: a unifying theory of aging? Epigenetics 2012, 7:680-8.

28. Scaffidi P, Misteli T: Lamin A-dependent nuclear defects in human aging. Science 2006, 312:1059-63.

29. Shumaker DK, Dechat T, Kohlmaier A, Adam SA, Bozovsky MR, Erdos MR, Eriksson M, Goldman AE, Khuon S, Collins FS, et al.: Mutant nuclear lamin A leads to progressive alterations of epigenetic control in premature aging. Proc. Natl. Acad. Sci. U. S. A. 2006, 103:8703-8.

30. Funayama R, Saito $\mathrm{M}$, Tanobe $\mathrm{H}$, Ishikawa F: Loss of linker histone $\mathbf{H 1}$ in cellular senescence. J. Cell Biol. 2006, 175:869-80.

31. Zhang R, Chen W, Adams PD: Molecular dissection of formation of senescence-associated heterochromatin foci. Mol. Cell. Biol. 2007, 27:2343-58.

32*. Chandra T, Kirschner K, Thuret J-Y, Pope BD, Ryba T, Newman S, Ahmed K, Samarajiwa SA, Salama R, Carroll T, et al.: Independence of repressive histone marks and chromatin compaction during senescent heterochromatic layer formation. Mol. Cell 2012, 47:203-14.

32* The authors describe SAHF as a multi-layered chromosomal architecture. Importantly, the authors show that SAHF heterochromatin is globally identical with heterochromatin in the nuclear periphery in growing cells and not de novo heterochromatin formation.

33. Zhang R, Poustovoitov M V, Ye X, Santos HA, Chen W, Daganzo SM, Erzberger JP, Serebriiskii IG, Canutescu AA, Dunbrack RL, et al.: Formation of MacroH2A-containing senescenceassociated heterochromatin foci and senescence driven by ASF1a and HIRA. Dev. Cell 2005, 8:19-30.

34. Chandra T, Narita M: High-order chromatin structure and the epigenome in SAHFs. Nucleus 2013, 4:23-8.

35. Bickmore WA, van Steensel B: Genome architecture: domain organization of interphase chromosomes. Cell 2013, 152:1270-84.

36**. Sadaie M, Salama R, Carroll T, Tomimatsu K, Chandra T, Young ARJ, Narita M, Pérez-Mancera $\mathrm{PA}$, Bennett $\mathrm{DC}$, Chong $\mathrm{H}$, et al.: Redistribution of the Lamin B1 genomic binding profile affects rearrangement of heterochromatic domains and SAHF formation during senescence. Genes Dev. 2013, 27:1800-8.

$36^{* *}$ This paper demonstrates that global Lamin B1 loss during senescence facilitates spatial reorganisation of chromatin (SAHF formation). In addition the authors find distinct regions gaining Lamin B1. These regions seem to be involved in gene repression.

37. Amendola $\mathrm{M}$, van Steensel $\mathrm{B}$ : Mechanisms and dynamics of nuclear lamina-genome interactions. Curr. Opin. Cell Biol. 2014, 28:61-8.

38. Dechat T, Pfleghaar K, Sengupta K, Shimi T, Shumaker DK, Solimando L, Goldman RD: Nuclear lamins: major factors in the structural organization and function of the nucleus and chromatin. Genes Dev. 2008, 22:832-53. 
39. Shimi T, Butin-Israeli V, Adam SA, Hamanaka RB, Goldman AE, Lucas CA, Shumaker DK, Kosak ST, Chandel NS, Goldman RD: The role of nuclear lamin B1 in cell proliferation and senescence. Genes Dev. 2011, 25:2579-93.

40. Shah PP, Donahue G, Otte GL, Capell BC, Nelson DM, Cao K, Aggarwala V, Cruickshanks HA, Rai TS, McBryan T, et al.: Lamin B1 depletion in senescent cells triggers large-scale changes in gene expression and the chromatin landscape. Genes Dev. 2013, 27:1787-99.

41**. Chandra T, Ewels PA, Schoenfelder S, Furlan-Magaril M, Wingett SW, Kirschner K, Thuret J-Y, Andrews S, Fraser P, Reik W: Global Reorganization of the Nuclear Landscape in Senescent Cells. Cell Rep. 2015, 10:471-83.

41** Using Hi-C technology, a sequence dependent loss of heterochromatin is detected in cellular senescence, mirroring the known loss of heterochromatin in Hutchinson -Gilford progeria syndrome. Moreover, senescence- specific clustering of heterochromatin reveals a second step during the formation of heterochromatic foci observed in cellular senescence.

42**. Dou Z, Xu C, Donahue G, Shimi T, Pan J-A, Zhu J, Ivanov A, Capell BC, Drake AM, Shah PP, et al.: Autophagy mediates degradation of nuclear lamina. Nature 2015, 527:105-9.

$42^{* *}$ This is a seminal report linking autophagy to the well established loss of nuclear Lamin_B1 during senescence.

43. Goldman RD, Shumaker DK, Erdos MR, Eriksson M, Goldman AE, Gordon LB, Gruenbaum Y, Khuon $S$, Mendez $M$, Varga $R$, et al.: Accumulation of mutant lamin A causes progressive changes in nuclear architecture in Hutchinson-Gilford progeria syndrome. Proc. Natl. Acad. Sci. U. S. A. 2004, 101:8963-8.

44. Gordon LB, Rothman FG, López-Otín C, Misteli T: Progeria: a paradigm for translational medicine. Cell 2014, 156:400-7.

45. Schreiber KH, Kennedy BK: When lamins go bad: nuclear structure and disease. Cell 2013, 152:1365-75.

46. Pegoraro G, Kubben N, Wickert U, Göhler H, Hoffmann K, Misteli T: Ageing-related chromatin defects through loss of the NURD complex. Nat. Cell Biol. 2009, 11:1261-7.

47. Lieberman-Aiden E, van Berkum NL, Williams L, Imakaev M, Ragoczy T, Telling A, Amit I, Lajoie $\mathrm{BR}$, Sabo PJ, Dorschner MO, et al.: Comprehensive mapping of long-range interactions reveals folding principles of the human genome. Science 2009, 326:289-93.

48. Meuleman W, Peric-Hupkes D, Kind J, Beaudry J-B, Pagie L, Kellis M, Reinders M, Wessels L, van Steensel $B$ : Constitutive nuclear lamina-genome interactions are highly conserved and associated with A/T-rich sequence. Genome Res. 2013, 23:270-80.

49*. McCord RP, Nazario-Toole A, Zhang H, Chines PS, Zhan Y, Erdos MR, Collins FS, Dekker J, Cao $\mathrm{K}$ : Correlated alterations in genome organization, histone methylation, and DNA-lamin A/C interactions in Hutchinson-Gilford progeria syndrome. Genome Res. 2013, 23:260-9. 
49* The authors show that Heterochromatin-lamina interactions are disturbed in HutchinsonGilford progeria syndrome. Hi-C experiments show a global loss of genome structure in HGPS cells.

50. Narita M, Narita M, Krizhanovsky V, Nuñez S, Chicas A, Hearn SA, Myers MP, Lowe SW: A novel role for high-mobility group a proteins in cellular senescence and heterochromatin formation. Cell 2006, 126:503-14.

51. Reeves R: High mobility group (HMG) proteins: Modulators of chromatin structure and DNA repair in mammalian cells. DNA Repair (Amst). 2015, doi:10.1016/j.dnarep.2015.09.015.

52. Corpet A, Stucki M: Chromatin maintenance and dynamics in senescence: a spotlight on SAHF formation and the epigenome of senescent cells. Chromosoma 2014, doi:10.1007/s00412-014-0469-6.

53. Rai TS, Adams PD: Lessons from senescence: Chromatin maintenance in non-proliferating cells. Biochim. Biophys. Acta 2012, 1819:322-31.

54. Swanson EC, Manning B, Zhang $\mathrm{H}$, Lawrence JB: Higher-order unfolding of satellite heterochromatin is a consistent and early event in cell senescence. J. Cell Biol. 2013, 203:929-42.

55. Swanson EC, Rapkin LM, Bazett-Jones DP, Lawrence JB: Unfolding the story of chromatin organization in senescent cells. Nucleus 2015, 6:254-60.

56. De Cecco M, Criscione SW, Peckham EJ, Hillenmeyer S, Hamm EA, Manivannan J, Peterson AL, Kreiling JA, Neretti N, Sedivy JM: Genomes of replicatively senescent cells undergo global epigenetic changes leading to gene silencing and activation of transposable elements. Aging Cell 2013, 12:247-56.

56** The authors show that heterochromatin reorganisation in replicative senescence leads to a more open chromatin state of repetitive elements leading to activation of retrotransposons .

57. De Cecco M, Criscione SW, Peterson AL, Neretti N, Sedivy JM, Kreiling JA: Transposable elements become active and mobile in the genomes of aging mammalian somatic tissues. Aging (Albany. NY). 2013, 5:867-83.

58. Van Meter M, Kashyap M, Rezazadeh S, Geneva AJ, Morello TD, Seluanov A, Gorbunova V: SIRT6 represses LINE1 retrotransposons by ribosylating KAP1 but this repression fails with stress and age. Nat. Commun. 2014, 5:5011.

59. Gorbunova V, Boeke JD, Helfand SL, Sedivy JM: Sleeping dogs of the genome. Science (80-. ). 2014, 346:1187-1188. 


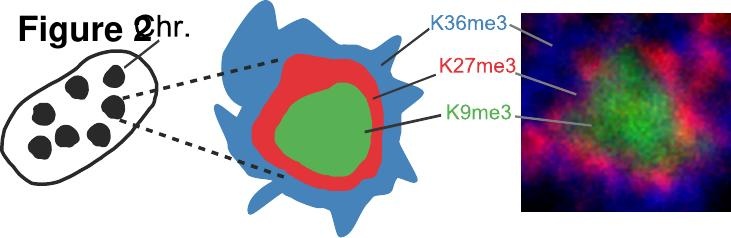


1. Step 2. Step

Acute/Chronic stress

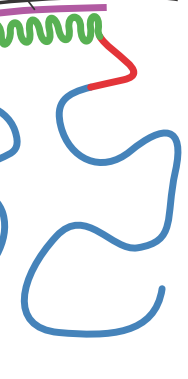

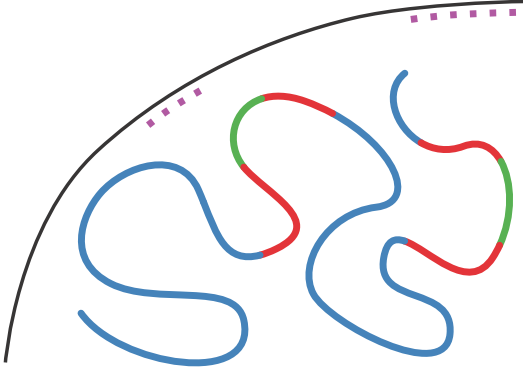

cHC relaxation
Acute stress/SAHF

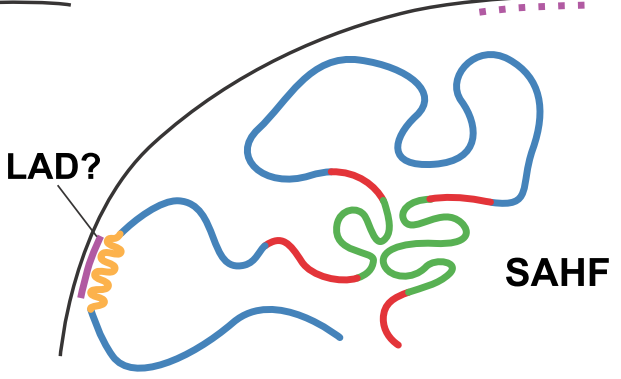

cHC clustering 
figrrenic and acute stress

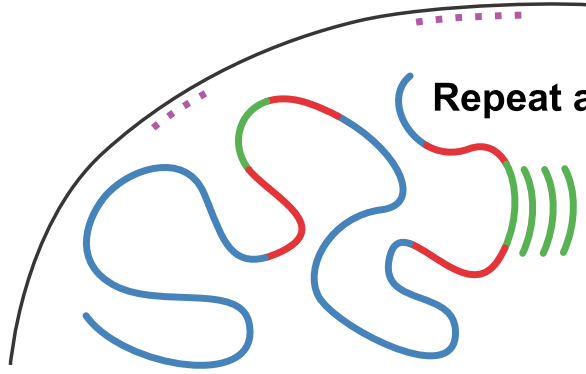

\section{Acute stress/SAHF}

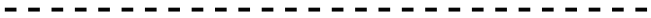

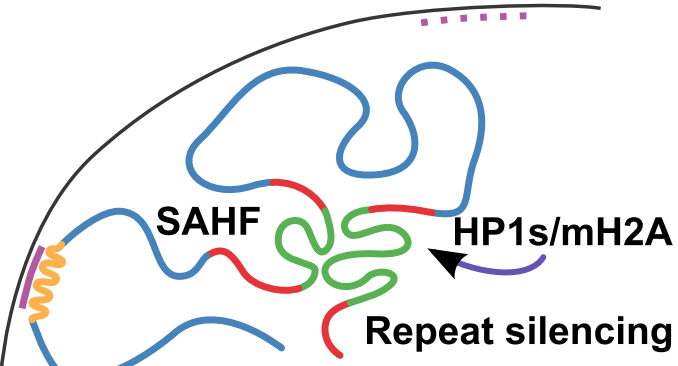

